\title{
Chapter 48 \\ PACAP as a Potential Biomarker: Alterations of PACAP Levels in Human Physiological and Pathological Conditions
}

\author{
Dora Reglodi, Zsuzsanna Helyes, Jozsef Nemeth, \\ Reka A. Vass, and Andrea Tamas
}

\begin{abstract}
Pituitary adenylate cyclase activating polypeptide (PACAP) is a neuropeptide with wide distribution and diverse effects. Its presence has not only been revealed in the nervous system and peripheral organs, but also in the blood and other biological fluids. Although PACAP is rapidly cleaved in the blood by peptidases, increasing number of evidence has been published in the last 10 years that show alterations of PACAP levels in different pathological conditions. The present review summarizes results obtained in the human blood and other fluids under normal, physiological and pathological conditions with clinical relevance, such as pregnancy and delivery, as well as in several diseases. Most clinical data have been obtained so far in neurological diseases, such as Alzheimer's disease, migraine, traumatic brain injury, multiple sclerosis, and cerebral hemorrhage, but some other diseases have also been shown to be accompanied by alterations of PACAP levels. These results indicate that the altered levels may have diagnostic and/or prognostic values in several diseases.
\end{abstract}

Keywords Serum $\bullet$ Plasma $\bullet$ RIA $\bullet$ ELISA $\bullet$ Biomarker $\bullet$ Neuropeptide

\footnotetext{
D. Reglodi $(\bowtie) \cdot$ R.A. Vass $\bullet$ A. Tamas

Department of Anatomy, MTA-PTE "Lendulet” PACAP Research Team,

University of Pecs, Szigeti u 12, Pecs 7624, Hungary

e-mail: reglodi@aok.pte.hu

Z. Helyes

Department of Pharmacology and Pharmacotherapy, University of Pecs, Pecs, Hungary

Janos Szentagothai Research Centre, MTA-PTE NAP B Chronic Pain Research Group,

University of Pecs, Pecs, Hungary

J. Nemeth

Department of Pharmacology and Pharmacotherapeutics, University of Debrecen,

Debrecen, Hungary
} 


\section{Introduction}

The pitfalls of neuropeptide measurements in biological samples are well known, and it is suggested to use neuropeptide immunoreactivity to describe what we measure with immunoassay techniques. This situation is further complicated in biological fluids: the stable tissue levels of neuropeptides change once the peptides enter the circulation and are broken down by peptidases. In addition, different pathological conditions and medications influence the levels and breakdown of peptides. In spite of difficulties and uncertainties, various peptides have been implicated as potential biomarkers in human diseases and their measurement in biological fluids have also helped to elucidate their involvement in several disease pathomechanisms [1].

Soon after the isolation of pituitary adenylate cyclase activating polypeptide (PACAP) by Miyata et al. [2], certain effects of PACAP in humans and its localization in different human tissues were described [3, 4]. The source of PACAP in the blood is not known with certainty. The most logical sources of circulating PACAP are tissues known to contain the highest level of PACAP, such as brain and endocrine glands. It has also been described that PACAP levels are 1.5 times higher in the mouse serum than in the plasma [5]. The authors suggested that platelets significantly contribute to circulating PACAP [5]. However, we do not know, for example in the case of the brain, how different barriers influence the passage of the peptide into the blood (see Chap. 25 by Banks). Another important issue to take into consideration is the short plasma elimination half-life of the peptide (5-10 min), similarly to other members of the VIP-secretin-glucagonGHRH peptide family [6].

PACAP levels have been measured in human blood plasma in several studies. In healthy volunteers, plasma PACAP levels showed no difference between radial artery, internal or external jugular vein or cubital vein [7] (Table 48.1). In addition to the baseline levels, it was shown that continuous infusion of PACAP caused rapid increase of plasma PACAP levels, and a rapid decrease after cessation of the infusion in a male patient, while inhalation of the peptide did not lead to increased plasma levels $[6,9]$. Subsequent reports also demonstrated that intravenous infusion in volunteers led to increased plasma levels [13]. PACAP is cleaved by dipeptidylpeptidase IV (DPP IV), which also cleaves several other peptides. The half-life of infused PACAP is estimated to be $3.5 \mathrm{~min}$ when infused to healthy young volunteers for $20 \mathrm{~min}$ [8], while others suggested a longer half-life of about 5-10 min [6] (Table 48.1).

Increasing evidence shows that plasma PACAP levels and PACAP in several other body fluids change under different physiological and pathological conditions and these levels may have diagnostic/prognostic values in certain diseases. The present review summarizes data obtained in human blood and other fluids under normal and pathological conditions (summarized in Tables 48.1, 48.2, and 48.3). 


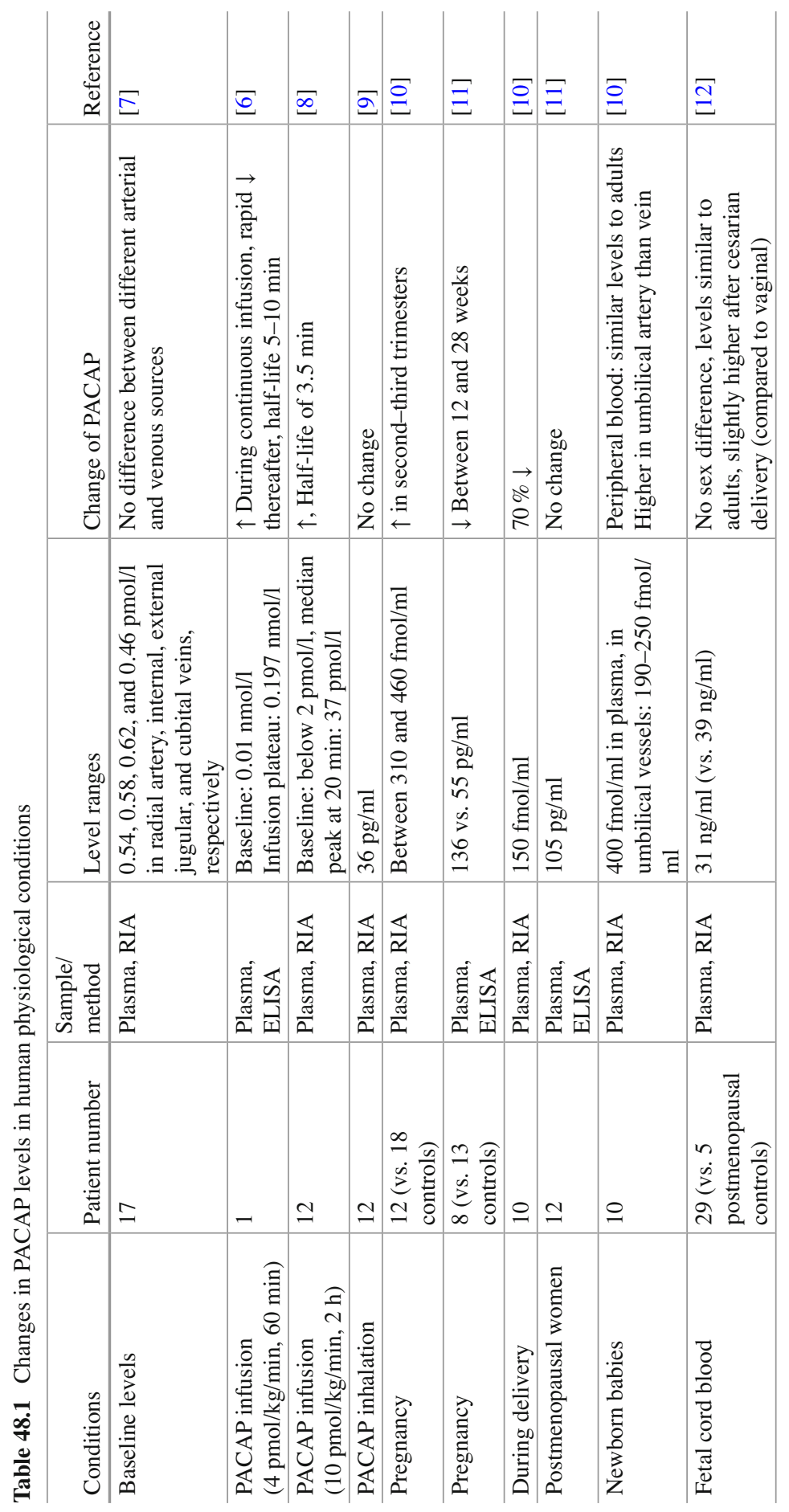




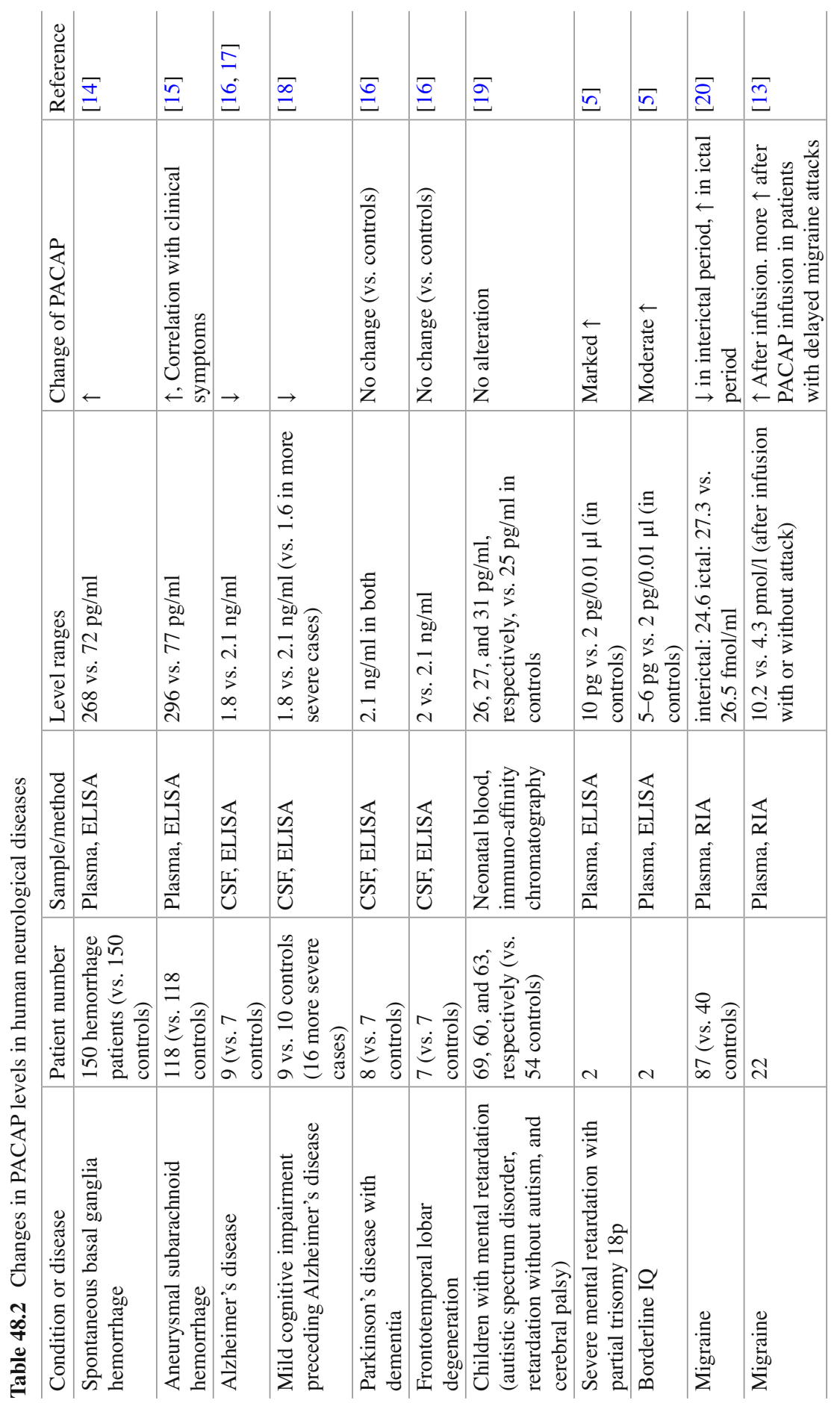




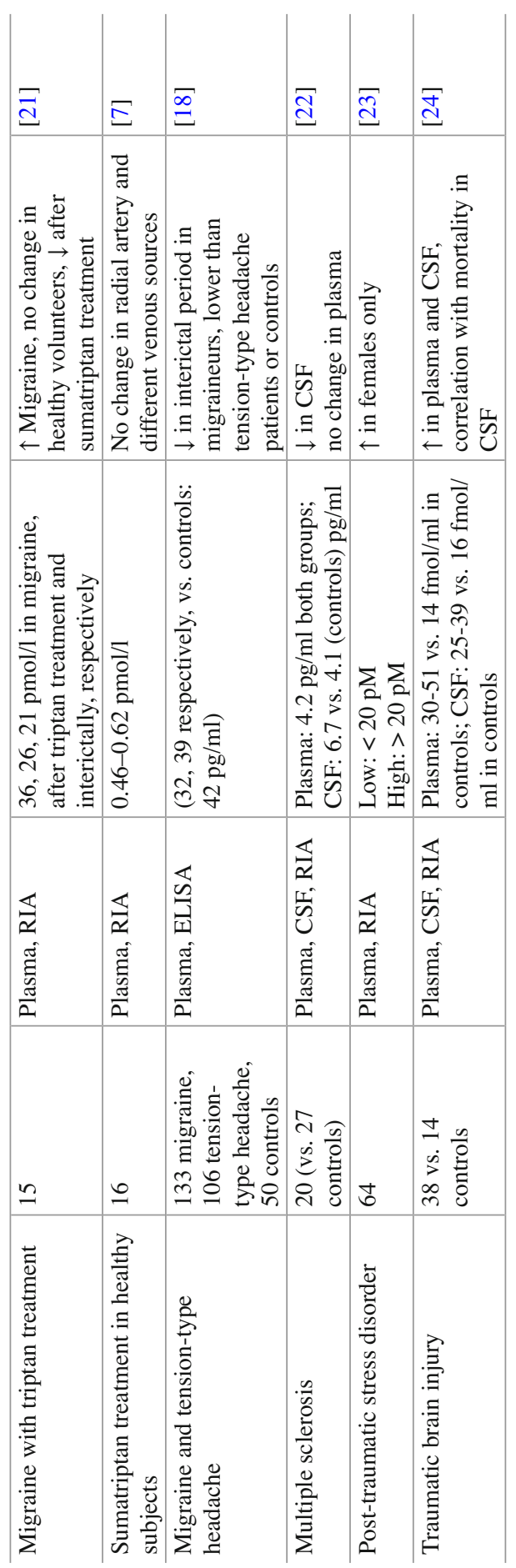


Table 48.3 Changes in PACAP levels in human non-neurological diseases

\begin{tabular}{|c|c|c|c|c|c|}
\hline Condition or disease & $\begin{array}{l}\text { Patient } \\
\text { number }\end{array}$ & $\begin{array}{l}\text { Sample/ } \\
\text { method }\end{array}$ & Level ranges & $\begin{array}{l}\text { Change of } \\
\text { PACAP }\end{array}$ & Reference \\
\hline $\begin{array}{l}\text { Chronic hepatitis B } \\
\text { patients under } \\
\text { antiviral therapy }\end{array}$ & $\begin{array}{l}25 \text { treated, } \\
22 \text { healthy } \\
\text { controls }\end{array}$ & $\begin{array}{l}\text { Plasma, } \\
\text { RIA }\end{array}$ & $\begin{array}{l}\text { Controls: } 65 \text {, } \\
\text { hepatitis: } 22 \\
\text { after treatment: } \\
49 \mathrm{pg} / \mathrm{ml}\end{array}$ & $\begin{array}{l}\downarrow \text { in hepatitis } \\
\text { patients, } \uparrow \text { after } \\
\text { antiviral therapy }\end{array}$ & [25] \\
\hline $\begin{array}{l}\text { Nephrotic syndrome } \\
\text { in children }\end{array}$ & $\begin{array}{l}28 \text { vs. } 10 \\
\text { controls }\end{array}$ & $\begin{array}{l}\text { Plasma, } \\
\text { urine, } \\
\text { Western } \\
\text { blot }\end{array}$ & $\begin{array}{l}\text { Results given } \\
\text { as } \% \text { of } \\
\text { controls, } \\
\text { absolute values } \\
\text { not available }\end{array}$ & $\begin{array}{l}\text { Plasma PACAP } \\
\downarrow \text {, urinary levels } \\
\uparrow \text { in nephrotic } \\
\text { states }\end{array}$ & [26] \\
\hline Nephrectomy & 3 & $\begin{array}{l}\text { Plasma, } \\
\text { Western } \\
\text { blot }\end{array}$ & As above & $\begin{array}{l}\uparrow \text { After } \\
\text { nephrectomy }\end{array}$ & [26] \\
\hline $\begin{array}{l}\text { Premature ovarian } \\
\text { failure }\end{array}$ & $\begin{array}{l}5 \text { (vs. } 13 \\
\text { controls) }\end{array}$ & $\begin{array}{l}\text { Plasma, } \\
\text { ELISA }\end{array}$ & $\begin{array}{l}58 \text { (vs. 136) } \\
\mathrm{pg} / \mathrm{ml}\end{array}$ & $\downarrow$ & [11] \\
\hline $\begin{array}{l}\text { Idiopathic } \\
\text { hypogonadotropic } \\
\text { hypogonadism }\end{array}$ & $\begin{array}{l}9 \text { (vs. } 13 \\
\text { controls) }\end{array}$ & $\begin{array}{l}\text { Plasma, } \\
\text { ELISA }\end{array}$ & $\begin{array}{l}92 \text { (vs. 136) } \\
\mathrm{pg} / \mathrm{ml}\end{array}$ & $\downarrow$ & [11] \\
\hline $\begin{array}{l}\text { Superovulation } \\
\text { treatment }\end{array}$ & 132 & $\begin{array}{l}\text { Follicular, } \\
\text { fluid RIA }\end{array}$ & $\begin{array}{l}143 \mathrm{fmol} / \mathrm{ml} \\
(28-690 \mathrm{fmol} / \\
\mathrm{ml}), \text { cutoff } \\
\text { value: } \\
290 \mathrm{fmol} / \mathrm{ml}\end{array}$ & $\begin{array}{l}\text { Correlation with } \\
\text { the number of } \\
\text { retrieved } \\
\text { oocytes: lower } \\
\text { levels } \\
\text { associated with } \\
\text { higher number } \\
\text { of oocytes }\end{array}$ & [27] \\
\hline
\end{tabular}

\section{PACAP Levels Under Physiological Conditions}

PACAP levels under normal circumstances are summarized in Table 48.1.

Early studies measured plasma PACAP levels in rats. A pioneer paper showed that the hypohyseal portal blood contained significantly higher PACAP immunoreactivity than the peripheral blood, with higher hypophyseal levels in females than in males ([28]; 50-100 pmol/1 vs. 23-24 pmol/l). This study proved that PACAP was released into the hypophyseal portal vessel blood and, therefore, can be regarded as a hypothalamic pituitary regulatory factor, with PACAP38 being the major form [28].

\section{Pregnancy and Delivery}

During pregnancy, PACAP levels show a moderate but significant elevation during the second and third trimesters compared to earlier gestational periods or nonpregnant women [10]. From the same volunteer population, during delivery, a significant, about $70 \%$, decrease was observed. Levels in the plasma of mothers returned to 
baseline 3 days after delivery. Plasma levels were also measured in the newborn babies of these volunteer mothers. It was found that the levels of PACAP were in the same range as in adults in the peripheral blood, but not in the blood obtained from the umbilical arteries, where PACAP level was lower [10]. The levels were higher in the umbilical arteries than in the umbilical vein. These findings indicate that during pregnancy PACAP is synthesized by the placenta or other maternal tissues in higher amounts, possibly necessary for the fetal and/or maternal tissue development. The sudden drop during delivery might indicate a role in the uteroplacental circulation and/or uterine contraction. The higher level in the umbilical artery (carrying venous blood from the fetus) than in the vein (carrying blood from the placenta to the fetus) indicates a fetal synthesis of the peptide [10].

Another study also investigated the level of PACAP in fetal cord blood and newborn babies [12]. The authors found no difference between male and female babies or between newborns and postmenopausal women between 64 and 83 years. It was also found that there was a slightly elevated level after caesarian delivery compared to vaginal birth, but there was no difference between PACAP levels in babies whose mothers progressed through labor or those who did not [12]. PACAP levels were unrelated to gestational age or Apgar score. The level of PACAP showed a slight increasing tendency with increasing birth weight. Based on the function of PACAP in stress adaptation and stress responses, it could be hypothesized that PACAP levels would differ in perinatal stress, but these data did not support this hypothesis.

In contrast to the above-mentioned results, Kanasaki and colleagues showed that plasma PACAP levels were lower in pregnant women compared to nonpregnant women [11]. PACAP levels were measured parallel with levels of luteinizing hormones, but no correlation was found between variations in PACAP and gonadotropin levels [11].

Related to pregnancy and childcare, other fluids also have to be mentioned: breast milk, amniotic fluid, and follicular fluid. Follicular fluid contained relatively stable levels of PACAP, with PACAP levels correlating with the number of retrieved oocytes during hyperstimulation treatment, the details of which are discussed below $[27,29]$. In the amniotic fluid, the presence of the unchanged PACAP molecule could not be detected by mass spectrometry, but RIA measurements confirmed PACAP-like immunoreactivity (LI) [29]. These results show that an altered or bound form of the peptide was present in the amniotic fluid, the significance of which still awaits further investigation. PACAP levels were markedly higher in the breast milk than in the plasma [30]. This topic is reviewed in a separate chapter (Chap. 49) of this book by Tamas et al.

\section{PACAP Levels Under Pathological Conditions}

\section{Neurological Diseases}

Changes in PACAP levels in neurological diseases are summarized in Table 48.2. 


\section{Alzheimer's Disease}

In postmortem human brains, Han and coworkers demonstrated a marked reduction in PACAP levels in several brain areas of Alzheimer's disease patients $[16,17]$. It was found that PACAP levels were reduced in the middle temporal gyrus, superior frontal gyrus, entorhinal cortex and primary visual cortex both at the mRNA and protein levels. Furthermore, the authors found that PACAP reduction was associated with pathological hallmarks of Alzheimer's disease: PACAP levels were reduced with higher amyloid plaque scores in the entorhinal cortex and superior temporal gyrus, but not in the primary visual cortex, a region spared in most cases of the disease. Levels were lower in advanced Braak stages than in moderate stages. Lower PACAP levels were measured in postmortem cisternal cerebrospinal fluid (CSF) of the same patients parallel with the reduced brain tissue levels [16]. Additionally, PACAP concentrations in patients with Alzheimer's disease strongly correlated with the dementia rating scores. The inverse correlation between PACAP and the pathological hallmarks of the disease suggests that PACAP is not only reduced in Alzheimer's disease, but also represents the severity of this pathology $[16,17]$. These data suggest that the strongly reduced neurotrophic effect of PACAP may be an important contributing factor to Alzheimer pathology. It supports the observations made in mouse models of Alzheimer's disease, where one of the downregulated genes important for neuronal survival was PACAP [31] and PACAP levels were strongly reduced in the brains of mutant mice resembling Alzheimer pathology [17].

In a subsequent study, the same research group showed that PACAP decline was associated with mild cognitive impairment preceding Alzheimer's disease in the above described brain areas [18]. It was found that PACAP levels were lower not only in the superior frontal gyrus and middle temporal gyrus but also in the CSF [18], although to a lesser extent than in fully developed Alzheimer's disease. Values were inversely correlated with the dementia rating score. These data suggest a progressive decline of PACAP levels in Alzheimer-related dementias. The expression level of the PAC1 receptor was also evaluated and it was shown to be upregulated in mild cognitive impairment, but not in fully developed Alzheimer's disease in the superior frontal gyrus [18]. This implies a transient, possibly compensatory upregulation of PAC1 receptor in mild impairment, which is lost at more progressed states [18]. The authors suggest that CSF PACAP could be a good candidate of disease progression marker given the close correlation with disease scores and the specificity, since no reduction was observed in other types of dementia (see below).

\section{Other Dementias}

An early study compared blood levels of several neuropeptides, including that of PACAP, in neonatal blood of children with autism spectrum disorder, mental retardation without autism and in children with cerebral palsy [19]. There was no difference in PACAP levels between the groups, in contrast to VIP, which showed higher 
levels in children with autism [19]. Han and coworkers measured CSF PACAP levels not only in Alzheimer's disease, but also in other types of dementia, such as Parkinson's disease with dementia and frontotemporal lobar degeneration. In contrast to the significant reduction observed in Alzheimer's disease, no reduction was observed in the CSF of other dementia patients postmortem [16]. In a family study, where members showed mental retardation, some displaying partial trisomy $18 \mathrm{p}$ with three copies of the PACAP gene [5]. It was found that two family members with severe mental retardation and accompanying other neurological problems had significantly higher level of PACAP, and two members with borderline IQs had moderately increased PACAP levels [5].

\section{Post-traumatic Stress Disorder}

Regarding the potential use of PACAP as a biomarker in post-traumatic stress disorder, a pioneer study was published in 2011 [23]. The authors found, for the first time, that there was a sex-specific association of PACAP blood levels with fear physiology and stress symptoms in females. Post-traumatic stress disorder symptoms significantly correlated with PACAP38 levels in females, but not in males, and the disease diagnosis was associated with PACAP levels in females, with higher PACAP38 found in the stress-disorder cohort. PACAP38 levels predicted differential response on the symptoms necessary to fulfill the criteria in females, like avoidance, hyperarousal, and reexperiencing [23]. They also found association with gene polymorphism of PACAP and PAC1 receptor, reinforced in a number of subsequent studies (further discussed in Chap. 45 of this book, by Ressler et al.).

\section{Acute Spontaneous Basal Ganglia Hemorrhage}

A recent study showed that plasma PACAP concentrations were significantly higher in patients after acute spontaneous basal ganglia hemorrhage than in healthy control subjects [14]. This study examined a high number of patients: 150 patients with stroke and 150 age- and gender-matched control individuals. In addition to elevated PACAP levels, a positive association was also shown between PACAP concentrations and neurological score, as well as with hematoma volume. Patients, who died within the first week after admission, had higher PACAP levels. A cutoff value was identified that predicted 1-week mortality with high sensitivity and specificity, and the predictive value was found to be similar to the neurological scores. Similarly, in patients with 6-month survival, elevated PACAP levels were found and it also predicted mortality with high sensitivity and specificity. Studying the overall survival, plasma PACAP concentration proved to be an independent predictor for overall survival similarly to scores and hematoma volumes. Overall survival times were significantly shorter in patients with high PACAP levels than those with low concentrations [14]. The authors suggested that PACAP could be a good prognostic predictor in hemorrhage patients and hypothesized that the elevated blood PACAP 
levels may reflect an overproduction of PACAP as a pathological response to the loss of neural tissue in the CNS and it might be associated with the neuroprotective effects of the neuropeptide. Another recent study has confirmed these findings in aneurysmal subarachnoid hemorrhage [15]. These authors examined 118 hemorrhage patients and compared them to 118 controls. Hemorrhage patients had significantly elevated PACAP levels on admission and plasma PACAP levels were independently associated with clinical severity scores. The neuropeptide was identified as an independent predictor of survival [15], suggesting that PACAP might be a potential prognostic biomarker of subarachnoid hemorrhage.

\section{Traumatic Brain Injury}

Our group demonstrated that PACAP could be reliably measured from the human CSF (25-80 fmol/ml) [24]. We measured PACAP levels in the plasma and CSF of patients who suffered severe traumatic brain injury. Patients were enrolled with a Glasgow Coma Scale $\leq 8$ on admission. We detected higher concentrations of PACAP in the CSF after traumatic brain injury and both plasma and CSF levels increased within the first 24-48 h after the injury. Thereafter, levels stayed high and did not increase further. In surviving patients, plasma and CSF levels were parallel, possibly indicating a damage in the blood-brain barrier. However, in patients, who died within the first week, plasma levels were almost double compared to levels of the CSF. There was no correlation with other clinical parameters [24]. These data show a relationship between first week mortality and plasma PACAP levels and support the possible hypothesis that PACAP levels are upregulated by the peri-injury areas, similarly to earlier immunohistochemical data from postmortem brains of traumatic brain injury patients [32]. However, similarly feasible explanation can be the relationship between the degree of blood-brain barrier injury and the elevated plasma PACAP levels.

\section{Multiple Sclerosis}

The role of PACAP in neuro-inflammatory processes was proposed on the basis of experimental data. Administration of PACAP suppressed experimental autoimmune encephalomyelitis, a model of multiple sclerosis [33]. Studies in mice supported these observations by showing that PACAP knockout mice exhibited exacerbated encephalomyelitis, thus, the endogenous production of the peptide protects against multiple sclerosis, probably by modulating regulatory $\mathrm{T}$ cells $[34,35]$. A clinical study investigated PACAP and VIP levels in the plasma and CSF of multiple sclerosis patients [22]. It was found that PACAP levels decreased in the CSF, while plasma PACAP levels did not change. These data suggest that PACAP may influence the course of multiple sclerosis. 


\section{Migraine}

The role of PACAP in migraine is evidenced both by animal and human studies. Due to the strong vasodilatory effect of PACAP and its sensitization function in the trigeminovascular system, PACAP is suggested to play an essential role in the induction of migraine [36]. Infusion of PACAP caused headache in healthy patients and migraine-like attacks in migraine patients [13, 37]. Animal studies supported these observations: PACAP38 was present in the trigeminal ganglion and caudal trigeminus nucleus, and plasma PACAP levels increased after electrical stimulation of the trigeminal ganglion [38] or after dural stimulation in cats [21]. PACAP knockout animals showed reduced responses to nitroglycerol [39].

As far as the plasma levels are concerned, Tuka et al. [20] found that plasma PACAP38 levels were significantly lower in the plasma of migraineurs during the interictal period than in healthy subjects, but elevated levels were shown during the ictal phase relative to the attack-free periods. In 21 migraineurs, samples were measured from the same subjects during attack and interictally. It was found that plasma PACAP38 levels were significantly higher during the ictal period. A negative correlation was observed between the interictal PACAP38 level and the disease duration [20]. Similarly to these data significantly lower plasma PACAP38-LI was detected in the inter-bout period of cluster headache patients than in healthy controls. Plasma PACAP38-LI was remarkably elevated during the attack compared to the attack-free period of patients (Tuka et al. unpublished observations). These results show that PACAP does not only play a role in the pathological mechanisms of different types of primary headaches, but its plasma levels are associated with the disease and show correlation with the migraine phases [40, 41]. Another recent study has further confirmed the significance of PACAP in the pathogenesis of migraine, rather than in tension-type headaches [18]. The authors confirmed significantly lower interictal levels of PACAP in migraineurs than in healthy controls or in tension-type headache patients. Unlike migraineurs, there was no difference in PACAP levels between samples from tension-type headache patients and healthy controls [18].

Another study also confirmed the elevation in PACAP levels during headache and lower levels between attacks [21]. In healthy volunteers, an anti-migraine medication (sumatriptan) did not lead to any change in circulating PACAP and VIP levels without trigeminovascular stimulation [7]. However, in migraine patients, sumatriptan treatment resulted in decreases in PACAP levels $1 \mathrm{~h}$ postinjection [21]. A recent study not only confirmed that PACAP38 infusion could trigger migraine attacks, but also found more increase in plasma PACAP38 levels in patients who reported delayed migraine attacks than in those patients who did not have headaches after PACAP infusion [13]. This observation indicates that migraine attacks were preceded by elevated plasma levels of PACAP38, similarly to the observation by others. The PACAP infusion-induced plasma elevations, in addition, indicate a de novo synthesis or release of the peptide. This human study raised interesting points 
on the source of elevated plasma PACAP levels: PACAP38 infusion to volunteers induced elevated plasma PACAP levels only in those patients who reported delayed migraine attacks. They found that after 20 min infusion, plasma levels in these patients were still elevated after $40 \mathrm{~min}$, which is beyond the $3.5 \mathrm{~min}$ half-life of the peptide. They suggest that the source of the peptide under normal conditions may represent neuronal release from PACAP-ergic nerve terminals. This can be triggered in migraine patients, which can result in further elevation [13].

\section{Other Diseases}

Changes in PACAP levels in non-neurological diseases are summarized in Table 48.3.

\section{Chronic Hepatitis}

PACAP levels were investigated in patients with chronic hepatitis under oral antiviral therapy (lamivudine) with RIA method [25]. The authors found no correlation between plasma PACAP38 levels and histological finding (liver biopsy), serum alanine amino transferase (ALT) level or serum hepatitis B virus -DNA level. However, they found that PACAP38 levels were significantly higher in healthy controls than in hepatitis B patients at baseline. This decrease was diminished after 52 weeks of antiviral therapy: PACAP levels significantly increased in treated patients. The authors suggested that the lower PACAP levels observed in hepatitis patients indicated the altered T1/T2 immune responses and related cytokine expression and 1 year of antiviral therapy could induce a marked amelioration also indicated by increased PACAP levels.

\section{Nephrotic Syndrome}

A recent study [26] has investigated PACAP levels in the plasma and urine in children with nephrotic syndrome. It was found that during the nephrotic state, PACAP was lost in the urine, compared with non-nephrotic state, when PACAP could not be reliably shown in the urine with Western blot. Parallel with the urinary loss, plasma PACAP levels decreased during these phases. Together with PACAP, the loss of its binding protein, ceruloplasmin, was also lost. These data indicate urinary loss of PACAP and its binding protein, thus resulting in a PACAP-deficient state. This was also accompanied by increased megakaryopoiesis and increased platelet aggregation [26]. Addition of PACAP inhibited the differentiation of hematopoetic stem cells into megakaryocytes during the nephrotic state, while addition of the plasma 
of congenital nephrotic patients during the nephrotic state directly promoted megakaryopoiesis when stem cells of healthy donors were used [26]. In a few patients, plasma PACAP levels were measured after bilateral nephrectomy, when gradually increasing levels were found together with decreased platelet count (with a strong inverse correlation in congenital nephrotic patients).

\section{Gynecological Diseases}

Lower levels of PACAP were found in patients with premature ovarian failure and in patients with idiopathic hypogonadotropic hypogonadism [11]. PACAP was also investigated in the follicular fluid of women undergoing superovulation treatment, where the presence of PACAP was confirmed with mass spectrometry analysis [27, 29]. Superovulation treatment is a usual procedure in controlled ovarian hyperstimulation during the in vitro fertilization procedure. After treatment, the number of harvested oocytes was recorded. All corresponding PACAP concentrations were below $290 \mathrm{fmol} / \mathrm{ml}$ in cases when the number of harvested oocytes exceeded 14 per patient, while in all cases above this cutoff value, the number of oocytes was below 14. Thus, lower levels of PACAP might correlate with a markedly higher number of ova retrieved and may predict a higher chance for ovarian hyperstimulation, a potentially life-threatening condition from excessive ovarian stimulation [27].

\section{Concluding Remarks: Future Perspectives and Limitations}

As summarized in the present review, increasing number of evidence suggests the potential clinical use of PACAP as a diagnostic and prognostic biomarker. Numerous data have been published in the last few years, showing that it is a rapidly developing, hot and promising topic. However, several limitations arise at the moment, which are briefly outlined below.

Presently we do not know where the PACAP measured in biological fluids, especially in the plasma, is exactly derived from. As already mentioned in the introductory part of this review, the most probable sources are the organs where PACAP occurs at high concentrations, like the nervous systems and the endocrine glands. In both physiological and pathological situations, PACAP levels in tissues can rapidly and drastically change and therefore, the levels measured under those conditions may represent PACAP from other sources as well. PACAP is upregulated in the central nervous system and peripheral tissues upon several injuries (rev. [42]). Serum PACAP levels have been shown to be increased after sensory nerve stimulation in rats $(0.38-0.8 \mathrm{fmol} / \mathrm{ml})$, or superior sagittal sinus stimulation in cats $(112$ $287 \mathrm{pmol} / \mathrm{l}$ ) indicating that levels can indeed rapidly change in response to physiological or pathological stimuli [21, 43]. In the skin of psoriatic patients, PACAP levels showed a 20-fold elevation [44]. Significantly higher tissue levels 
were also shown in cardiac tissue obtained from patients with ischemic heart disease [45], while lower levels/downregulation were found in other diseases: in lung and colon cancer samples [45]. In another recent study, no alterations in PACAP-LI between healthy and tumoral samples from the urinary bladder and testis were detected, but lower PACAP38-LI was found in kidney tumors compared with healthy tissue samples, and higher PACAP27-LI in prostatic cancer compared to samples from benign prostatic hyperplasia [46]. These data indicate that PACAP levels of different tissue samples are altered under pathological conditions suggesting a potential role of PACAP on tumor growth [46]. Reduction of PACAPcontaining nerve fibers was described in a few conditions, such as in women with polycystic ovaries [47]. To what extent these human tissue changes are reflected in alterations of serum PACAP levels is not known at the moment, but animal data suggest that acute local changes can cause changes of circulating PACAP as well.

Several recent studies have shown alterations in human plasma and other fluids in relation to certain diseases as detailed above. However, the question whether PACAP alterations are consequences or contributing factors of the disease still remains open [17]. One possibility is that the triggering cause of a disease leads to PACAP decline resulting in a higher vulnerability of the cells, as the neuroprotective effect of PACAP is reduced. However, it is equally possible that the PACAP deficit-for an unknown reason-predisposes neurons and other cells to certain pathological changes [17].

Several studies suggest that future biomarkers will be used in panels including several markers of different sensitivity and specificity [48]. Whether PACAP will indeed be a biomarker is a question to be answered by future studies, but based on our current knowledge it seems that PACAP is a good candidate for certain diseases and can be included in a panel of biomarkers after mapping the conditions where PACAP alterations are present or absent.

One main limitation of the presently available data pool is that we cannot compare results from different laboratories using different methods [1]. Even the same method, but from different manufacturers, can yield non-comparable results. Different PACAP antisera developed for immunohistochemistry, RIA or ELISA are most of the times not highly specific, are directed against different parts of the peptide or the prepro-peptide, in which case other cleavage products might also be bound by the antibody. Some authors interpret their results as PACAPimmunoreactivity even though the antiserum was developed against prepro-PACAP, in other cases the fact that the antiserum was produced against prepro-PACAP is not even mentioned in the paper, it only turns out by studying the manufacturer's description. Due to the cleavage by DPPIV, inactive PACAP3-38 and PACAP5-38 are yielded. The different antibodies used for detection of PACAP may also recognize these inactive forms, so we do not know at the moment whether PACAPimmunoreactivity measured in the blood plasma/serum always indicates the active peptide. As pointed out by Edvinsson et al. [1], this mixture of peptide fragments also belong to the physiological picture and, therefore, should not be neglected. If we use the term "PACAP- or PACAP-LI", it can cover all these forms. Revealing changes in PACAP-like immunoreactivity can yield important results as for the biological and pathological significance of the peptide. In addition, PACAP binds to 
plasma proteins, and the best documented carrier candidate among them is ceruloplasmin [49]. It has not been clearly demonstrated whether the measured PACAP reflects total, free, and/or protein-bound PACAP.

Several further technical limitations limit the comparison of results between groups. One of them is the way how human samples are obtained and stored. As PACAP is quickly degraded by DPPIV, peptidase inhibitors are required to use. However, this is not always indicated in the papers. Some data indicate that PACAP27 is much more resistant to DPPIV degradation than PACAP38 and therefore has a much longer half-life [50]. Thus, the ratio between the two peptides may also change with time, which is not taken into consideration by any clinical report. The different anticoagulants used can lead to different values, as shown by Freson et al. [5]. The time passed between sample collection and measurement is also critical. Some authors have described PACAP levels in postmortem CSF samples [16], which obviously cannot be compared to fresh samples taken from patients. Another issue that needs to be taken into consideration is the range within which PACAP levels change. In some studies very small changes are significant and authors draw final conclusions, while in other studies much bigger alterations are not significant and therefore are interpreted as no change. In some studies the sample sizes are very high, with more than a hundred involved patients, while in other studies, only a few patients are included in one group and even case studies are available with a single patient's values.

One further limitation is the lack of a fast and sensitive analytical method that is able to measure PACAP from small sample volumes. In spite of all these factors that limit the comparison of data, the alterations within one study can yield valuable results showing changes of PACAP levels, which can not only lead to biomarker development but can also reveal important functions of PACAP in physiological and pathological processes. In order to obtain a solid proof of PACAP as a biomarker, multicenter, controlled studies will be necessary in the future, but the growing body of evidence for the diagnostic and prognostic value of the peptide summarized in the current review justify these efforts.

Acknowledgements This study was supported by the following grants: OTKA K104984, 115874, 119759, Arimura Foundation, TAMOP 4.2.4.A/2-11-1-2012-0001 "National Excellence Program", New National Excellence Program (UNKP), Janos Bolyai Research Scholarship of Hungarian Academy of Sciences, MTA-PTE "Lendulet" Program, PTE AOK KA Research Grant, and the National Brain Research Programmes A and B (KTIA_NAP_13-2014-0022 and KTIA_13_NAPA-III/5, Research site ID number: 888819, Hungary). This work is dedicated to the 650th anniversary of the University of Pecs.

\section{References}

1. Edvinsson L, Ekman R, Goadsby PJ. Measurement of vasoactive neuropeptides in biological materials: problems and pitfalls from 30 years of experience and novel future approaches. Cephalalgia. 2010;30:761-6.

2. Miyata A, Arimura A, Dahl RR, Minamino N, Uehara A, Jiang L, et al. Isolation of a novel 38 residue-hypothalamic polypeptide which stimulates adenylate cyclase in pituitary cells. Biochem Biophys Res Commun. 1989;164:567-74. 
3. Palkovits M, Somogyvari-Vigh A, Arimura A. Concentrations of pituitary adenylate cyclase activating polypeptide (PACAP) in human brain nuclei. Brain Res. 1995;699:116-20.

4. Warren JB, Cockcroft JR, Larkin SW, Kajekar R, Macrae A, Ghatei MA, et al. Pituitary adenylate cyclase activating polypeptide is a potent vasodilator in humans. J Cardiovasc Pharmacol. 1992;20:83-7.

5. Freson K, Hashimoto H, Thys C, Wittevrongel C, Danloy S, Morita Y, et al. The pituitary adenylate cyclase-activating polypeptide is a physiological inhibitor of platelet activation. J Clin Invest. 2004;113:905-12.

6. Li M, Maderdrut JL, Lertora JJ, Batuman V. Intravenous infusion of pituitary adenylate cyclase-activating polypeptide (PACAP) in a patient with multiple myeloma and myeloma kidney: a case study. Peptides. 2007;28:1891-5.

7. Hansen JM, Fahrenkrug J, Petersen J, Wienecke T, Olsen KS, Ashina M. Vasoactive intestinal peptide (VIP) and pituitary adenylate cyclase-activating polypeptide (PACAP) in the circulation after sumatriptan. Scand J Pain. 2013;4:211-6.

8. Birk S, Sitarz JT, Petersen KA, Oturai PS, Kruuse C, Fahrenkrug J, et al. The effect of intravenous PACAP38 on cerebral hemodynamics in healthy volunteers. Regul Pept. 2007;140:185-91.

9. Doberer D, Gschwandtner M, Mosgoeller W, Bieglmayer C, Heinzl H, Petkov V. Pulmonary and systemic effects of inhaled PACAP38 in healthy male subjects. Eur J Clin Invest. 2007;37:665-72.

10. Reglodi D, Gyarmati J, Ertl T, Borzsei R, Bodis J, Tamas A, et al. Alterations of pituitary adenylate cyclase-activating polypeptide-like immunoreactivity in the human plasma during pregnancy and after birth. J Endocrinol Invest. 2010;33:443-5.

11. Kanasaki H, Purwana IN, Oride A, Mijiddorj T, Sukhbaatar U, Miyazaki K. Circulating kisspeptin and pituitary adenylate cyclase-activating polypeptide (PACAP) do not correlate with gonadotropin serum levels. Gynecol Endocrinol. 2013;29:583-7.

12. Winters SJ, King JC, Brees CK, Moore Jr JP. Pituitary adenylate cyclase-activating polypeptide (PACAP) in fetal cord blood. Early Hum Dev. 2014;90:451-3.

13. Amin FM, Hougaard A, Schytz HW, Asghar MS, Lundholm E, Parvaiz AI, et al. Investigation of the pathophysiological mechanisms of migraine attacks induced by pituitary adenylate cyclase-activating polypeptide-38. Brain. 2014;137:779-94.

14. Ma BQ, Zhang M, Ba L. Plasma pituitary adenylate cyclase-activating polypeptide concentrations and mortality after acute spontaneous basal ganglia hemorrhage. Clin Chim Acta. 2015;439:102-6.

15. Jiang L, Wang WH, Dong XQ, Yu WH, Du Q, Yang DB, et al. The change of plasma pituitary adenylate cyclase-activating polypeptide levels after aneurysmal subarachnoid hemorrhage. Acta Neurol Scand. 2016;197:35-42.

16. Han P, Liang W, Baxter LC, Yin J, Tang Z, Beach TG, et al. Pituitary adenylate cyclaseactivating polypeptide is reduced in Alzheimer disease. Neurology. 2014;82:1724-8.

17. Han P, Tang Z, Yin J, Maalouf M, Beach TG, Reiman EM, et al. Pituitary adenylate cyclaseactivating polypeptide protects against $\beta$-amyloid toxicity. Neurobiol Aging. 2014;35:2064-71.

18. Han P, Caselli RJ, Baxter L, Serrano G, Yin J, Beach TG, et al. Association of pituitary adenylate cyclase-activating polypeptide with cognitive decline in mild cognitive impairment due to Alzheimer disease. JAMA Neurol. 2015;72:333-9.

19. Nelson KB, Grether JK, Croen LA, Dambrosia JM, Dickens BF, Jelliffe LL, et al. Neuropeptides and neurotrophins in neonatal blood of children with autism or mental retardation. Ann Neurol. 2001;49:597-606.

20. Tuka B, Helyes Z, Markovics A, Bagoly T, Szolcsanyi J, et al. Alterations in PACAP38-like immunoreactivity in the plasma during ictal and interictal periods of migraine patients. Cephalalgia. 2013;33:1085-95.

21. Zagami AS, Edvinsson L, Goadsby PJ. Pituitary adenylate cyclase activating polypeptide and migraine. Ann Clin Transl Neurol. 2014;1:1036-40.

22. Baranowska-Bik A, Kochanowski J, Uchman D, Wolinska-Witort E, Kalisz M, Martynska L, et al. Vasoactive intestinal peptide (VIP) and pituitary adenylate cyclase activating polypeptide (PACAP) in humans with multiple sclerosis. J Neuroimmunol. 2013;263:159-61. 
23. Ressler KJ, Mercer KB, Bradley B, Jovanovic T, Mahan A, Kerley K, et al. Post-traumatic stress disorder is associated with PACAP and the PAC1 receptor. Nature. 2011;470:492-7.

24. Bukovics P, Czeiter E, Amrein K, Kovacs N, Pal J, Tamas A, et al. Changes of PACAP level in cerebrospinal fluid and plasma of patients with severe traumatic brain injury. Peptides. 2014;60:18-22.

25. Elefsiniotis IS, Ketikoglou I, Kafiri G, Pantazis KD, Moulakakis A, Mavrogiannis C. Plasma pituitary adenylate cyclase activating polypeptide (PACAP) levels in chronic hepatitis B patients under lamivudine treatment. Eur J Gastroenterol Hepatol. 2003;15:1209-16.

26. Eneman B, Freson K, van den Heuvel L, van Hoyweghen E, Collard L, Vande Walle J, et al. Pituitary adenylate cyclase-activating polypeptide deficiency associated with increased platelet count and aggregability in nephrotic syndrome. J Thromb Haemost. 2015;13:755-67.

27. Koppan M, Varnagy A, Reglodi D, Brubel R, Nemeth J, Tamas A, et al. Correlation between oocyte number and follicular fluid concentration of pituitary adenylate cyclase-activating polypeptide (PACAP) in women after superovulation treatment. J Mol Neurosci. 2012;48: $617-22$.

28. Dow RC, Bennie J, Fink G. Pituitary adenylate cyclase-activating peptide-38 (PACAP)-38 is released into hypophysial portal blood in the normal male and female rat. $\mathrm{J}$ Endocrinol. 1994;142:R1-4.

29. Brubel R, Reglodi D, Jambor E, Koppan M, Varnagy A, Biro Z, et al. Investigation of pituitary adenylate cyclase activating polypeptide in human gynecological and other biological fluids by using MALDI TOF mass spectrometry. J Mass Spectrom. 2011;46:189-94.

30. Borzsei R, Mark L, Tamas A, Bagoly T, Bay C, Csanaky K, et al. Presence of pituitary adenylate cyclase activating polypeptide-38 in human plasma and milk. Eur $\mathrm{J}$ Endocrinol. 2009;160:561-5.

31. Wu ZL, Ciallella JR, Flood DG, O'Kane TM, Bozyczko-Coyne D, Savage MJ. Comparative analysis of cortical gene expression in mouse models of Alzheimer's disease. Neurobiol Aging. 2006;27:377-86.

32. van Landeghem FK, Weiss T, Oehmichen M, von Deimling A. Cellular localization of pituitary adenylate cyclase-activating peptide (PACAP) following traumatic brain injury in humans. Acta Neuropathol. 2007;113:683-93.

33. Kato H, Ito A, Kawanokuchi J, Jin S, Mizuno T, Ojika K, et al. Pituitary adenylate cyclaseactivating polypeptide (PACAP) ameliorates experimental autoimmune encephalomyelitis by suppressing the functions of antigen presenting cells. Mult Scler. 2004;10:651-9.

34. Tan YV, Abad C, Wang Y, Lopez R, Waschek JA. VPAC2 (vasoactive intestinal peptide receptor type 2) receptor deficient mice develop exacerbated experimental autoimmune encephalomyelitis with increased Th1/Th17 and reduced Th2/Treg responses. Brain Behav Immun. 2015;44:167-75.

35. Tan YV, Waschek JA. Targeting VIP and PACAP receptor signalling: new therapeutic strategies in multiple sclerosis. ASN Neuro. 2011;3(4):e00065.

36. Vecsei L, Tuka B, Tajti J. Role of PACAP in migraine headaches. Brain. 2014;137:650-1.

37. Schytz HW, Birk S, Wienecke T, Kruuse C, Olesen J, Ashina M. PACAP38 induces migrainelike attacks in patients with migraine without aura. Brain. 2009;132:16-25.

38. Tuka B, Helyes Z, Markovics A, Bagoly T, Nemeth J, Mark L, et al. Peripheral and central alterations of pituitary adenylate cyclase activating polypeptide-like immunoreactivity in the rat in response to activation of the trigeminovascular system. Peptides. 2012;33:307-16.

39. Markovics A, Kormos V, Gaszner B, Lashgarara A, Szoke E, Sandor K, et al. Pituitary adenylate cyclase-activating polypeptide plays a key role in nitroglycerol-induced trigeminovascular activation in mice. Neurobiol Dis. 2012;45:633-44.

40. Tajti J, Tuka B, Botz B, Helyes Z, Vecsei L. Role of pituitary adenylate cyclase-activating polypeptide in nociception and migraine. CNS Neurol Disord Drug Targets. 2015;14:540-53.

41. Tajti J, Szok D, Majlath Z, Tuka B, Csati A, Vecsei L. Migraine and neuropeptides. Neuropeptides. 2015;52:19-30.

42. Somogyvari-Vigh A, Reglodi D. Pituitary adenylate cyclase activating polypeptide: a potential neuroprotective peptide. Curr Pharm Des. 2004;10:2861-89. Review. 
43. Nemeth J, Reglodi D, Pozsgai G, Szabo A, Elekes K, Pinter E, et al. Effect of pituitary adenylate cyclase activating polypeptide- 38 on sensory neuropeptide release and neurogenic inflammation in rats and mice. Neuroscience. 2006;143:223-30.

44. Steinhoff M, McGregor GP, Radleff-Schlimme A, Steinhoff A, Jarry H, Schmidt WE. Identification of pituitary adenylate cyclase activating polypeptide (PACAP) and PACAP type 1 receptor in human skin: expression of PACAP38 is increased in patients with psoriasis. Regul Pept. 1999;80:49-55.

45. Szanto Z, Sarszegi Z, Reglodi D, Nemeth J, Szabadfi K, Kiss P, et al. PACAP immunoreactivity in human malignant tumor samples and cardiac diseases. J Mol Neurosci. 2012;48:667-73.

46. Tamas A, Javorhazy A, Reglodi D, Sarlos DP, Banyai D, Semjen D, et al. Examination of PACAP-like immunoreactivity in urogenital tumor samples. J Mol Neurosci. 2015;2015: PMID:26454744.

47. Wojtkiewicz J, Jana B, Kozłowska A, Crayton R, Majewski M, Zalecki M, et al. Innervation pattern of polycystic ovaries in the women. J Chem Neuroanat. 2014;6162:147-52.

48. Chan MK, Gottschalk MG, Haenisch F, Tomasik J, Ruland T, Rahmoune H, et al. Applications of blood-based protein biomarker strategies in the study of psychiatric disorders. Prog Neurobiol. 2014;122:45-72.

49. Tams JW, Johnsen AH, Fahrenkrug J. Identification of pituitary adenylate cyclase-activating polypeptide1-38-binding factor in human plasma, as ceruloplasmin. Biochem J. 1999;341: 271-6.

50. Bourgault S, Vaudry D, Botia B, Couvineau A, Laburthe M, Vaudry H, et al. Novel stable PACAP analogs with potent activity towards the PAC1 receptor. Peptides. 2008;29:919-32. 